\title{
Semantically Aware Bayesian Network Model for Actionable Knowledge Discovery in Linked Data
}

\author{
Hasanein Alharbi, Mohamad Saraee \\ ${ }^{1}$ School of Computing, Science and Engineering, University of Salford-Manchester, \\ Greater Manchester, England M5 4WT \\ H.Y.M.AlHarbi@edu.Salford.ac.uk,m.saraee@salford.ac.uk \\ Abstract.The majority of the convential mining \\ algorithms treat the mining process as an isolated \\ data-driven procedure and overlook the semantic of \\ the targeted data. As a result, the generated \\ patterns are abundant and end users cannot act upon \\ them seamlessly. Furthermore, interdisciplinary \\ knowledge could no be obtained from domain-specifi \\ silo of data. \\ The emergence of Linked Data (LD) as a new model for \\ knowledge representation, which intertwines data with \\ its semantics, has introduced new opportunities for \\ data miners. Accordingly, this paper proposes a \\ Semantic-Aware Bayesian network (BN) model, which \\ exploits the semantic aspectes of the LD structure.It \\ integrates five semantic relations in the mining \\ process. \\ In contrast to the exisiting mining algorithms, the \\ proposed model do not transform the original format \\ of the LD set. So, it not only accomodates the \\ semantic aspects in LD, but also caters to the need of \\ connecting different data-sets from different \\ domains. \\ Keywords: Linked Data (LD). Actionable Knowledge \\ Discovery (AKD). Bayesian Network (BN).
}

1 Introduction

The term Data Mining (DM) refers to methods that aim to extract useful information and knowledge from data. Fayyad et al. have defined these methods as the non-trivial process of identifying valid, novel, potentially useful and ultimately understandable patterns in a database [1,2].

Despite the fact that the ultimate goal of DM is to identify useful and understandable patterns, the existing mining algorithms are confined to generating frequent patterns and do not illustrate how to act upon them. Accordingly, the concept of Actionable Knowledge Discovery (AKD) has been introduced to overcome the shortages on traditional mining algorithms. The goal of AKD techniques is to bridge the gap between the output of the current mining algorithms and the needs of the real life applications $[3,4,5]$. 
This gap has appeared as a result of two major drawbacks, namely, quantity and quality; the former states that the generated patterns are abundant while the latter indicates that they cannot be integrated seamlessly into the business domain [3], [6]. Upon further investigation, it appears these drawbacks have been caused as a result of viewing the mining process as data driven trial and error practices and ignoring the surrounding knowledge [3], [7]. Consequently, the mining philosophy has faced a paradigm shift from a data-centered to a knowledge-centered process, which aims to integrate the surrounding knowledge such as data intelligence into the mining process [3], [8]. Even though the data intelligence could be represented using various techniques, recently LD introduced a new technique to intertwine the data and its semantics in one package. Coupling the data with its semantics not only brings new opportunities for data miners but also raised some challenges; for example, how to identified the interesting transactions in heterogeneous data sets, which has been built based on the description logic and used the triple (subject-predicate-object) format $[9,10]$. To this end, this paper proposes a semantic-aware Bayesian network model, which exploits the semantic nature of LD and implicitly accommodates the data intelligence in the mining process. The proposed approach consists of the following steps:

1. Convert the original LD file into BN, which preserves the semantics of the LD file.

2. Initializes the Conditional Probability Tables (CPT's) with default values.

3. Calculate set of probabilistic constraints using the concept of Maximum a Posterior estimation (MAP) in such a way that it reflects the semantic relations between nodes in the constructed BN.

4. Approximate the CPT's initial values to comply with the set constraints calculated in the second step using the concept of Iterative Proportional Fitting Procedure (IPFP).

The contributions of this paper are twofold. Firstly, the model integrates five semantic relations in the mining process, namely, equivalent to, complement of, disjoint with, intersection of and union of. Secondly, it does not change the original format of LD set and consequently, it caters for the need of linking various data-sets from multidisciplinary domains using the design principles of LD. The proposed model tested using five sets of synthesis data and the initial results are promising.

The remainder of this paper is organized as follows: In section 2 the notion of converting $\mathrm{LD}$ file into $\mathrm{BN}$ is explained in detail, while the probabilistic constraints estimation methods are illustrated in section 3. IPFP is briefly discussed in the section 4, and section 5 discusses in detail the empirical implementation and the initial results. Finally, the paper is concluded in section 6.

\section{Bayesian Network Topology Construction}

BayesOWL consists of a set of construction rules, which convert ontology files into BN directed acyclic graphs (DAG), which preserve the semantics of the original ontology file [11,12]. Likewise, the model proposed in this paper follows the same rules to convert the given LD file into BN DAG.

The construction of BayesOWL graph has two main phases. In the first phase, the BayesOWL graph structure is built and the associated CPT's are initialized with de- 
fault values. Then, the integration of the given probabilistic constraints is implemented in the second phase $[13,14]$.

The process of construction BN DAG from the given ontology file is governed by a set of rules. The conventions underpinning these rules can be summarized in the following points [12], [15]:

1. Every primitive or defined class is mapped into binary variables.

2. Connect each parent superclass with its child subclass by an arc.

3. For each concept class $C$ is defined as the intersection of a set of classes $C_{i}=\left\{C_{1}\right.$, ....., $\mathrm{C}_{\mathrm{n}}$ \}; a subnet is created in such a way that there is a link from $\mathrm{C}$ and each class in the set $\mathrm{C}_{\mathrm{i}}$ toward the class $\mathrm{C}$. Furthermore, there is a link from $\mathrm{C}$ and each class in the set $\mathrm{C}_{\mathrm{i}}$ moves toward a logical node called LNodeIntersection. Figure 1.a depicts the creation of the intersection subnet.

4. For each concept class $C$ is defined as the union of classes $C_{i}=\left\{C_{1}, \ldots . ., C_{n}\right\}$; a subnet is created in such a way that there is a link from $C$ to each class in the set $C_{i}$. Furthermore, there is a link from $\mathrm{C}$ and each class in the set $C_{\mathrm{i}}$ moves toward a logical node called LNodeUnion. Figure 1.b illustrates the creation of the union subnet.

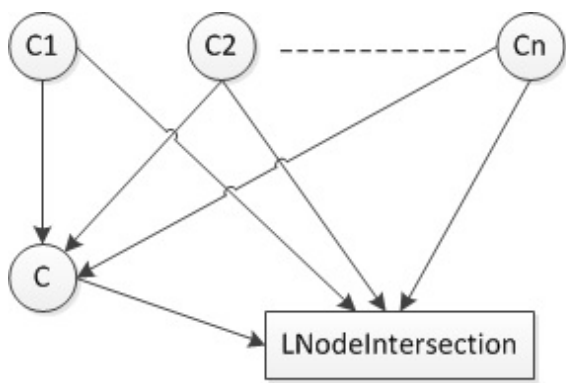

$-a-$

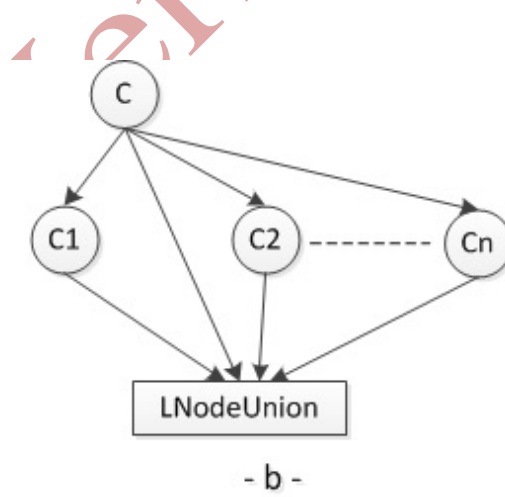

$-b-$

Fig. 1. LNodeIntersection \& LNodeUnion [11]

5. For each two concept classes $C_{1}$ and $C_{2}$ are defined as complements of, equivalent to, disjoint with each other logical nodes (LNodeComplement, LNodeEquivalent, LNodeDisjoint) are created, which take two input links from $C_{1}$ and $C_{2}$. Figures 2.a, 2.b and 2.c depict the creation process for LNodeComplement, LNodeEquivalent and LNodeDisjoint respectively.
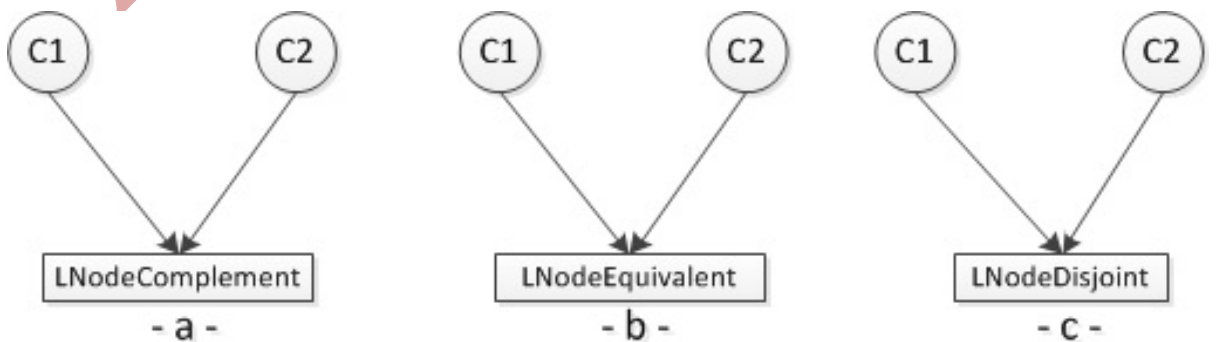

Fig. 2. LNodeComplement \& LNodeEquivalent \& LNodeDisjoint [11] 
It clearly can be seen that the generated DAG contains two types of nodes, namely, regular nodes, which represent classes and logical nodes, which show the logical relation among classes [11], [15]. The combination of these two types of nodes forms the structure of the BN.

The second component of $\mathrm{BN}$ is the conditional probability table. As discussed in the previous paragraph, the generated DAG contains two types of nodes, logical and regular. Consequently, the CPT's for each type should be calculated. The following subsection explains in detail the CPT's calculation process [11], [16, 15].

\subsection{CPT's calculation for logical nodes}

It has been stated that the generated DAG caters for five different types of logical nodes, which are associated with five logical operations in ontology. The CPT for each logical node is determined by its logical relations. The following subsections explain the CPT creation process for each logical node.

\section{CPT creation for Complement of logical relation.}

The complement relation between two concepts classes $C_{1}$ and $C_{2}$ is true IFF $c 1 \overline{c 2} v \overline{c 1} c 2$ is true. Table 1 describes the CPT for complement of relation.

CPT creation for Disjoint with logical relation.

The disjoint with relation between two concept classes $C_{1}$ and $C_{2}$ is true IFF $c 1 \overline{c 2} v \overline{c 1} c 2 v \overline{c 1 c 2}$ is true. Table 2 describes the CPT for disjoint with relation.

\section{CPT creation for Equivalent to logical relation.}

The equivalent to relation between two concept classes $C_{1}$ and $C_{2}$ is true IFF $c 1 c 2 v \overline{c 1 c 2}$ is true. Table 3 describes the CPT for equivalent of relation.

Table 1. CPT for LnodeComplementOF [11]

\begin{tabular}{|c|c|c|c|}
\hline $\mathrm{C}_{1}$ & $\mathrm{C}_{2}$ & True & False \\
\hline $\mathrm{T}$ & $\mathrm{T}$ & 0 & 1 \\
\hline $\mathrm{T}$ & $\mathrm{F}$ & 1 & 0 \\
\hline $\mathrm{F}$ & $\mathrm{T}$ & 1 & 0 \\
\hline $\mathrm{F}$ & $\mathrm{F}$ & 0 & 1 \\
\hline
\end{tabular}

Table 2. CPT for LnodeDisjointWith [11]

\begin{tabular}{|c|c|c|c|}
\hline $\mathrm{C}_{1}$ & $\mathrm{C}_{2}$ & True & False \\
\hline $\mathrm{T}$ & $\mathrm{T}$ & 0 & 1 \\
\hline $\mathrm{T}$ & $\mathrm{F}$ & 1 & 0 \\
\hline $\mathrm{F}$ & $\mathrm{T}$ & 1 & 0 \\
\hline $\mathrm{F}$ & $\mathrm{F}$ & 1 & 0 \\
\hline
\end{tabular}

Table 3. CPT for LnodeEquivalentTo [11]

\begin{tabular}{|c|c|c|c|}
\hline $\mathrm{C}_{1}$ & $\mathrm{C}_{2}$ & True & False \\
\hline $\mathrm{T}$ & $\mathrm{T}$ & 1 & 0 \\
\hline $\mathrm{T}$ & $\mathrm{F}$ & 0 & 1 \\
\hline $\mathrm{F}$ & $\mathrm{T}$ & 0 & 1 \\
\hline $\mathrm{F}$ & $\mathrm{F}$ & 1 & 0 \\
\hline
\end{tabular}

CPT creation for Intersection of logical relation.

The class $C$, which is the intersection of $\mathrm{C}_{1}$ and $\mathrm{C}_{2}$ is true IFF $c c 1 c 2 \sqrt{c c 1} c 2 \vee \bar{c} c 1 \overline{c 2} \mathrm{v} \overline{c c 1 c 2}$ is true. Table 4 describes the CPT for the intersection of relation.

CPT creation for Union of logical relation.

The class C, which is the union of C1 and C2 is true IFF $c c 1 c 2 \vee c \overline{c 1} c 2 \vee c c 1 \overline{c 2} v \overline{c c 1 c 2}$ is true. Table 5 describes the CPT for the union of relation. 
Table 4. CPT for IntersectionOf [11]

\begin{tabular}{|c|c|c|c|c|}
\hline $\mathrm{C}$ & $\mathrm{C}_{1}$ & $\mathrm{C}_{2}$ & True & False \\
\hline $\mathrm{T}$ & $\mathrm{T}$ & $\mathrm{T}$ & 1 & 0 \\
\hline $\mathrm{T}$ & $\mathrm{T}$ & $\mathrm{F}$ & 0 & 1 \\
\hline $\mathrm{T}$ & $\mathrm{F}$ & $\mathrm{T}$ & 0 & 1 \\
\hline $\mathrm{T}$ & $\mathrm{F}$ & $\mathrm{F}$ & 1 & 0 \\
\hline $\mathrm{F}$ & $\mathrm{T}$ & $\mathrm{T}$ & 0 & 1 \\
\hline $\mathrm{F}$ & $\mathrm{T}$ & $\mathrm{F}$ & 1 & 0 \\
\hline $\mathrm{F}$ & $\mathrm{F}$ & $\mathrm{T}$ & 0 & 1 \\
\hline $\mathrm{F}$ & $\mathrm{F}$ & $\mathrm{F}$ & 1 & 0 \\
\hline
\end{tabular}

Table 5. CPT for UnionOF [11]

\begin{tabular}{|c|c|c|c|c|}
\hline $\mathrm{C}$ & $\mathrm{C}_{1}$ & $\mathrm{C}_{2}$ & True & False \\
\hline $\mathrm{T}$ & $\mathrm{T}$ & $\mathrm{T}$ & 1 & 0 \\
\hline $\mathrm{T}$ & $\mathrm{T}$ & $\mathrm{F}$ & 0 & 1 \\
\hline $\mathrm{T}$ & $\mathrm{F}$ & $\mathrm{T}$ & 1 & 0 \\
\hline $\mathrm{T}$ & $\mathrm{F}$ & $\mathrm{F}$ & 0 & 1 \\
\hline $\mathrm{F}$ & $\mathrm{T}$ & $\mathrm{T}$ & 1 & 0 \\
\hline $\mathrm{F}$ & $\mathrm{T}$ & $\mathrm{F}$ & 0 & 1 \\
\hline $\mathrm{F}$ & $\mathrm{F}$ & $\mathrm{T}$ & 0 & 1 \\
\hline $\mathrm{F}$ & $\mathrm{F}$ & $\mathrm{F}$ & 1 & 0 \\
\hline
\end{tabular}

\subsection{CPT calculation for regular nodes}

The CPT's for regular nodes are computed by applying the Bayesian theorem as follows. $\mathrm{P}\left(\mathrm{C} \mid \pi_{\mathrm{C}}\right)$ where $\mathrm{C}$ is the class of the regular node and $\pi_{\mathrm{c}}$ is the set of its parents. The $\mathrm{P}\left(\mathrm{C}=\right.$ True $\left.\mid \pi_{\mathrm{c}}\right)=0$ if any of its parents are false. Hence, the probability for any regular class $\mathrm{C}$ is calculated only when all of its parents are in true status. This scenario is denoted as $\mathrm{P}\left(\mathrm{C} \mid \pi_{\mathrm{c}}^{+}\right)$where $\pi_{\mathrm{c}}^{+}$represents the set of parent classes in true status. This method is used to calculate the probability when probabilistic data is available. Otherwise, a default value (0.5) is assigned based on equation (1) [11], [13].

$$
\mathrm{P}\left(\mathrm{C}=\text { True } \mid \pi_{c}^{+}\right)=\mathrm{P}\left(\mathrm{C}=\text { False } \mid \pi_{c}^{+}\right)=0.5 \text {. }
$$

\section{Probabilistic Constraints Estimation}

The process of converting OWL (i.e. LD) file into BN consists of two phases. In the first phase the BN structure is constructed and the associated CPT's are initialized with default values. Then, the probabilistic constraints are integrated in the second phase. Hereafter, the process of probabilistic constraints estimation is covered in detail.

It has been argued that the two main approaches for probabilistic estimation in BN are the Maximum Likelihood Estimation (MLE) and Bayesian estimation $[17,18,19]$. Thus, these two approaches are discussed in the next subsections.

\subsection{Maximum Likelihood Estimation (MLE)}

MLE aims to find the value of $\Theta$, which quantifies the maximum probability of the incoming event. In a data-set $\mathrm{D}$, which consists of $\mathrm{n}$ instances of binominal random variable $X$ then MLE aims to estimate the maximum likelihood of occurrence for $n+1$ incoming event [17], [20].

It is assumed that, the random variable $\mathrm{X}$ represents the event of flipping a thumbtack, which has two possible outcomes, Head and Tail. Furthermore, the observed data consist of 5 observations, 3 of which are Heads and 2 Tails. Therefore, the MLE for the incoming event $n+1$ for $\mathrm{X}=$ Head is:

$$
\mathrm{MLE}_{\mathrm{x}=\text { Head }}=\frac{\mathrm{X}=\text { Head }}{\mathrm{X}=\text { Head }+\mathrm{X}=\text { Tail }}+\frac{3}{3+2}=0.6
$$

It can be seen that the likelihood function is maximized by dividing the number of correct trials over the total number of trials. Although MLE has various advantages, it also has some limitations. For example, the size of the observed data-set has no effect 
in the estimation process. Additionally, MLE does not take the prior knowledge into consideration and entirely relies on the observed data. Therefore, Bayesian method, which integrates the prior knowledge in the estimation process, has been introduced [17,18], [20].

\subsection{Maximum a Posterior Estimation (MAP)}

An alternative method for parameters estimation, which injects the prior knowledge in the form of prior distribution in the estimation process, is MAP. MLE aims to maximize the likelihood function. Likewise, MAP aims to maximize the posterior of $\Theta$ given in the observed data. This hypothesis can be formalized in the following equation [17], [20]:

$$
\widehat{\theta} M A P=\operatorname{argmax} \mathrm{P}(\Theta \mid \mathrm{d})
$$

Equation (3) could be reformulated using Bayes rule.

$$
\begin{gathered}
\widehat{\theta} M A P=\operatorname{argmax} \frac{\mathrm{p}(\mathrm{d} \mid \theta) p(\theta)}{\mathrm{p}(\mathrm{d})} \text { Where } \mathrm{p}(\mathrm{d}) \neq f(\Theta) \\
\widehat{\theta} M A P=\operatorname{argmax}(\log p(d \mid \Theta)+\log p(\Theta))
\end{gathered}
$$

Equation (5) shows that the posterior probability $p(\Theta \mid d)$ is Beta distribution, which is obtained by summing the likelihood in form of Bernoulli distribution and prior knowledge in form of Beta distribution. Hence, the posterior probability is Beta distribution with $(\alpha+r)$ correct trials out of $(\alpha+\beta+n)$ total number of trials. Accordingly, the prior and posterior statistics for Beta distribution could be summarized in the following table [19], [21].

Table 6. Prior and posterior statistics for beta distribution [19]

\begin{tabular}{|l|l|l|}
\hline \multicolumn{1}{|c|}{ Statistics } & \multicolumn{1}{|c|}{ Prior } & \multicolumn{1}{c|}{ Posterior } \\
\hline Law & $\operatorname{Beta}(\alpha, \beta)$ & $\operatorname{Beta}(\alpha+\mathrm{r}, \beta+(\mathrm{n}-\mathrm{r}))$ \\
\hline Mean & $\alpha / \alpha+\beta$ & $\alpha+\mathrm{r} / \alpha+\beta+\mathrm{n}$ \\
\hline Mode & $\alpha-1 / \alpha+\beta-2$ & $\alpha+\mathrm{r}-1 / \alpha+\beta+\mathrm{n}-2$ \\
\hline variance & $\alpha \beta /(\alpha+\beta) 2+(\alpha+\beta+1)$ & $(\alpha+\mathrm{r})(\beta+\mathrm{n}-\mathrm{r}) /(\alpha+\beta+\mathrm{n}) 2+(\alpha+\beta+\mathrm{n}+1)$ \\
\hline
\end{tabular}

\section{Iterative Proportional Fitting Procedure (IPFP)}

The concept of Iterative Proportional Fitting Procedure (IPFP) was first introduced by Deming and Stephan in 1940. It used to estimate the probability in contingency table, which is subject to given marginal constraints. In 2000, Cramer proposed an extension to the traditional IPFP to accommodate the conditional probability constraints. In fact, the statistical application for probability models with marginal and conditional distributions are comprehensive, such as, Bayesian statistic, contingency table, longlinear models etc. [22, 23].

This paper is concerned with the capability of IPFP to approximate a set of probability tables according to a given set of marginal and conditional probabilistic constraints. The full mathematical and theoretical background of IPFP is beyond the scope of this paper. The reader may refer to the following reference [24] for comprehensive studies on IPFP.

\section{$5 \quad$ Empirical Implementation and Initial Results}

The proposed model has been tested using five sets of synthesized data. Each data-set highlighted the significance of one logical relation. The process sequence is designed 
in such a way that it shows the effects of integrating the logical relations between random variables in $\mathrm{BN}$. As a matter of fact, it compares the output of the Loopy Believe Propagation (LBP) inference algorithm using two different sets of probabilistic constraints. The first set ignores the logical relations between random variables while the second set accommodates these relations. Consequently, each data-set is processed in two different ways and the final results have been compared. Figure (3) shows the process sequence and the actions taken in each approach.

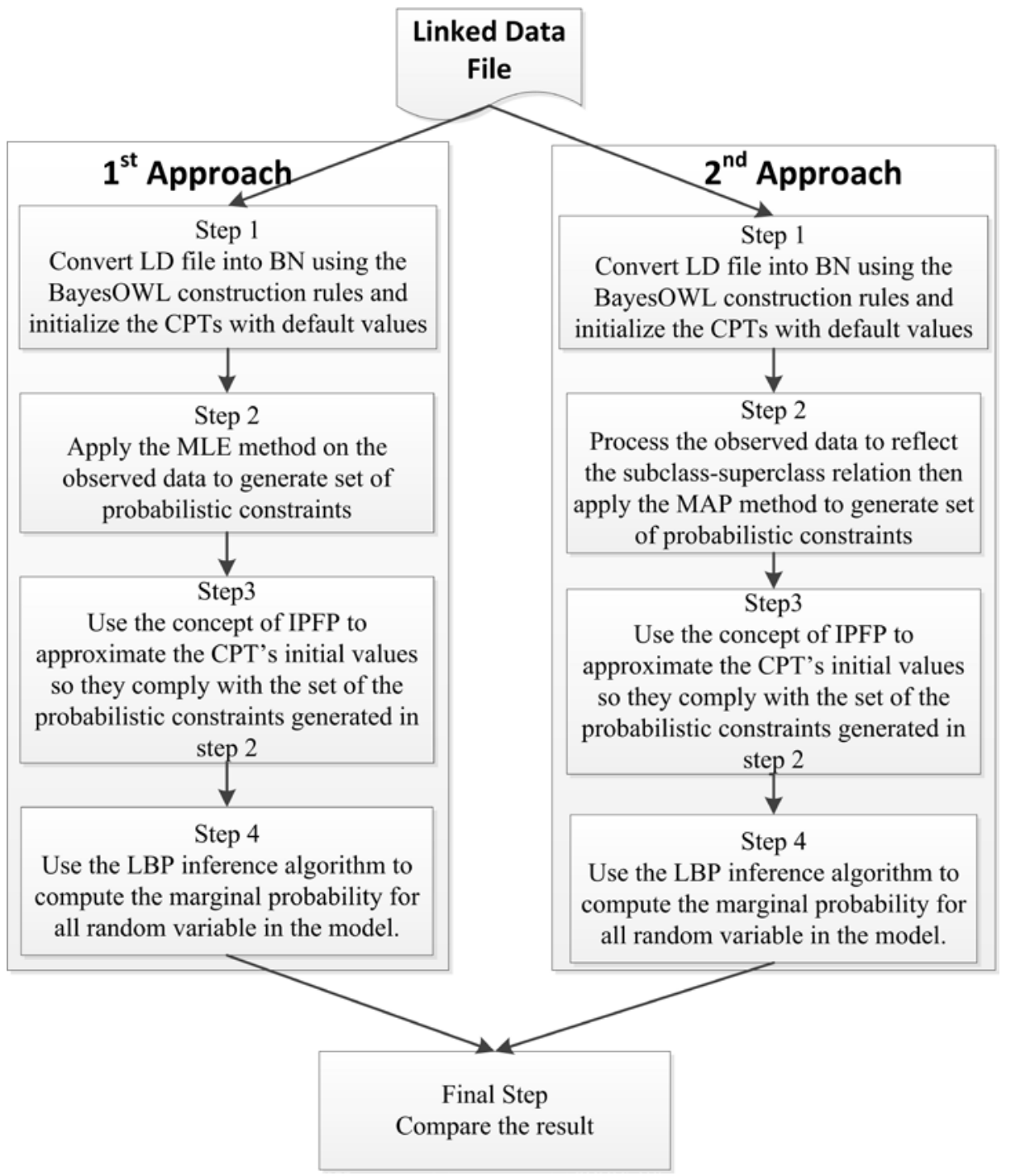

Fig. 3. Process sequence 


\subsection{Preliminaries and Notations}

Figure 3 shows that each data-set has been process in two differnt approaches. The first approach ignor the semantic relations between random variables while the second approach integrate these relations. Table (7) explains in details the steps on each approach.

Table 7. Proces walkthrough

\begin{tabular}{|c|c|c|}
\hline Steps & 1st approach & 2nd approach \\
\hline 1 & \multicolumn{2}{|c|}{$\begin{array}{l}\text { Convert the LD file into BN using the construction rules explained in section } 2 \\
\text { and initialized the associated CPT's with default value (i.e. 0.5). }\end{array}$} \\
\hline 2 & $\begin{array}{l}\text { Calculate the probabilistic con- } \\
\text { straints using the MLE techniques } \\
\text { base on the following equation. } \\
M L E=\mathrm{M} 1 \text { /M1+M2 } \\
\text { Where: } \\
\text { - M1 is the number of true trial. } \\
\text { - M2 is the number of false trial. }\end{array}$ & $\begin{array}{l}\text { - Process the o observed data in such a } \\
\text { way that reflect the superclass-subclass } \\
\text { relations. In simple words, an instance } \\
\text { of each class is observed as an instance } \\
\text { of its superclass. } \\
\text { - Calculate the probabilistic constraints } \\
\text { using the MAP techniques based on the } \\
\text { following equation. } \\
M A P=(\mathrm{M} 1+\alpha-1) /(\mathrm{M} 1+\alpha+\mathrm{M} 2+\beta-2)(7) \\
\text { Where: } \\
\text { - M1 is the number of true trial. } \\
\text { - } \mathrm{M} 2 \text { is the number of false trial. } \\
\text { - } \beta \text { is the number of true trail in the se- } \\
\text { semantic data-set. }\end{array}$ \\
\hline 3 & \multicolumn{2}{|c|}{$\begin{array}{l}\text { Approximate the CPT's initial values using the concept of IPFP and the set of } \\
\text { constrains calculated in step } 2 \text {. }\end{array}$} \\
\hline 4 & \multicolumn{2}{|c|}{$\begin{array}{l}\text { Calculate the marginal probability for all random variable using the LBP infer- } \\
\text { ence algorithm. }\end{array}$} \\
\hline
\end{tabular}

Table (7) clearly shows that steps 1, 3 and 4 are identical. However, the calculation of the probabilistic constraints in step 2 is differ. Hence, the input for steps 3 and 4 is changed accordingly. As a matter of fact, $\alpha$ and $\beta$ hayperparameters in equation 7 were used to cater for the semantic relation other than the superclass-subclass relations. These parameters utilized to inject the semantic relation into the estimation process.

\subsection{Examples}

This section is divided into five subsections. Each subsection gives an example, which covers one logical relation. Furthermore, the generated result is compared and discussed. 


\section{Example One (Equivalent of logical relation).}

Figure 4.a indicates that class D is equivalent to class E. Hence, any instance of D is an instance of $\mathrm{E}$ and vice versa. Furthermore, it shows the superclass-subclass relations between variables (i.e. classes) in example one. Table 8 illustrates the calculation of the probabilistic constraints for the $1^{\text {st }}$ and $2^{\text {nd }}$ approach.

Table 8. Example one $1^{\text {st }}$ approach Vs $2^{\text {nd }}$ approach constraints calculations

\begin{tabular}{|c|c|c|c|c|c|}
\hline \multicolumn{2}{|c|}{ Example 1 } & \multicolumn{2}{|c|}{$1^{\text {st }}$ approach } & \multicolumn{2}{|c|}{$2^{\text {nd }}$ approach } \\
\hline Class & Subclasses & $\begin{array}{c}\text { Observed } \\
\text { data }\end{array}$ & $\begin{array}{c}\text { MLE } \\
(\mathrm{M} 1 / \mathrm{M} 1+\mathrm{M} 2)\end{array}$ & $\begin{array}{c}\text { Processed } \\
\text { observed } \\
\text { data }\end{array}$ & $\begin{array}{c}\text { MAP } \\
(\mathrm{M} 1+\alpha-1) / \\
(\mathrm{M} 1+\alpha+\mathrm{M} 2+\beta-2)\end{array}$ \\
\hline $\mathrm{R}$ & $\{\mathrm{A}, \mathrm{B}\}$ & 1 & $1 /(1+15)=0.063$ & 16 & $(16+1-1) /(16+1+0+1-2)=1.000$ \\
\hline $\mathrm{A}$ & $\{\mathrm{C}, \mathrm{D}\}$ & 1 & $1 /(1+15)=0.063$ & 10 & $(10+1-1) /(10+1+6+1-2)=0.625$ \\
\hline $\mathrm{B}$ & $\{\mathrm{E}, \mathrm{F}\}$ & 1 & $1 /(1+15)=0.063$ & 5 & $(5+1-1) /(5+1+11+1-2)=0.313$ \\
\hline $\mathrm{C}$ & \{\} & 2 & $2 /(2+14)=0.125$ & 2 & $(2+1-1) /(2+1+14+1-2)=0.125$ \\
\hline $\mathrm{D}$ & \{\} & 7 & $7 /(7+9)=0.438$ & 7 & $(7+9-1) /(7+9+9+7-2)=0.500$ \\
\hline $\mathrm{E}$ & \{\} & 2 & $2 /(2+14)=0.125$ & 2 & $(2+9-1) /(2+9+14+7-2)=0.333$ \\
\hline $\mathrm{F}$ & \{\} & 2 & $2 /(2+14)=0.125$ & 2 & $(2+1-1) /(2+1+14+1-2)=0.125$ \\
\hline
\end{tabular}

\section{Discussions.}

Table 8 shows how the observed data has been process to reflect the subclasssuperclass relations. Additionally, it explains how the hayperparameters have been used to inject the semantic relations into the estimation process. For example, the value of $\alpha$ hayperparameter for class D and E is equal to 9 , which is the sum of the $\mathrm{D}$ and $\mathrm{E}$ instance because they are equivalent.

Figure 4.b clearly shows that the results of the second technique (i.e MAP) have reflected the semantic relations between the random variables in example one. For example, the marginal probabilities for classes D and E, which were involved in the equivalent semantic relation, have increased compared to their marginal probabilities in the first approach. Likewise, the marginal probabilities for all classes involved in superclass-subclass relation were increased accordingly.

\section{Example two (disjoint with logical relation).}

Figure 4.c indicates that class A is disjoint with class B. The semantic interpretation of this relation is that an instance of class A and its subclasses could not be an instance of class B and its subclasses and vice versa. Additionally, it shows the superclass-subclass relations between classed in example two. The observed data processing and the calculation for the probabilistic constraints with and without integrating the semantic relations are explained in table 9. 
Table 9. Example two $1^{\text {st }}$ approach Vs $2^{\text {nd }}$ approach constraints calculations

\begin{tabular}{|c|c|c|c|c|c|}
\hline \multicolumn{2}{|c|}{ Example 2} & \multicolumn{2}{|c|}{$1^{\text {st }}$ approach } & \multicolumn{2}{c|}{$2^{\text {nd }}$ approach } \\
\hline Class & $\begin{array}{c}\text { Sub- } \\
\text { classes }\end{array}$ & $\begin{array}{c}\text { Observed } \\
\text { data }\end{array}$ & $\begin{array}{c}\text { MLE } \\
(\mathrm{M} 1 / \mathrm{M} 1+\mathrm{M} 2)\end{array}$ & $\begin{array}{c}\text { Processed } \\
\text { observed } \\
\text { data }\end{array}$ & $\begin{array}{c}\text { MAP } \\
(\mathrm{M} 1+\alpha-1) /(\mathrm{M} 1+\alpha+\mathrm{M} 2+\beta-2)\end{array}$ \\
\hline $\mathrm{R}$ & $\{\mathrm{A}, \mathrm{B}\}$ & 1 & $1 /(1+24)=0.040$ & 25 & $(25+1-1) /(25+1+0+1-2)=1.000$ \\
\hline $\mathrm{A}$ & $\{\mathrm{C}, \mathrm{D}\}$ & 3 & $3 /(3+22)=0.120$ & 9 & $(9+9-1) /(9+9+16+1-2)=0.515$ \\
\hline $\mathrm{B}$ & $\{\mathrm{E}\}$ & 5 & $5 /(5+20)=0.200$ & 15 & $(15+15-1) /(15+15+10+1-2)=0.744$ \\
\hline $\mathrm{C}$ & \{\} & 2 & $2 /(2+23)=0.080$ & 2 & $(2+2-1) /(2+2+23+8-2)=0.091$ \\
\hline $\mathrm{D}$ & \{\} & 4 & $4 /(4+21)=0.160$ & 4 & $(4+4-1) /(4+4+21+6-2)=0.212$ \\
\hline $\mathrm{E}$ & $\{\mathrm{F}, \mathrm{H}\}$ & 6 & $6 /(6+19)=0.240$ & 10 & $(10+10-1) /(10+10+15+6-2)=0.487$ \\
\hline $\mathrm{F}$ & \{\} & 2 & $2 /(2+23)=0.080$ & 2 & $(2+2-1) /(2+2+23+14-2)=0.077$ \\
\hline $\mathrm{H}$ & \{\} & 2 & $2 /(2+23)=0.080$ & 2 & $(2+2-1) /(2+2+23+14-2)=0.077$ \\
\hline
\end{tabular}

Discussion.

Table 9 illustrates how the observed data has been processed to reflect the superclasssubclass relations. Furthermore, it shows how the value of $\beta$ changes to accommodate the disjoint relations. For example, for class $C$ the value of $\beta$ is equal to 8 , which means, the possibility that an instance belong to class $C$ workspace but not an instance of class $\mathrm{C}$ it only could be an instance of class R, A or D because class A is disjoint with class B and its subclasses.

Figure 4.d compares between the marginal probabilities for all random variables in exampole two with and without considering the semantic relations between these variables. It can be seen that the marginal probability for classes A, B and their subclasses have accomodate the disjoint semantic relation.

\section{Example three (Complement of logical relation).}

This example simulates the complement of semantic relation. Figure 4.e shows that class A is complement of class B. The semantic effect of this relation is that an instance of class A and its subclasses cannot be an instance of class B and its subclasses. Likewise, an instance of class B and its subclasses cannot be an instance of class A and its subclasses. Table 10 below explains the observed data processing step and the constraints estimation with and without injecting the semantic relation between classes in example three.

Table 10. Example three $1^{\text {st }}$ approach Vs $2^{\text {nd }}$ approach constraints calculations

\begin{tabular}{|c|c|c|c|c|c|}
\hline \multicolumn{2}{|c|}{ Example 3 } & \multicolumn{2}{|c|}{$1^{\text {st }}$ approach } & \multicolumn{2}{c|}{$2^{\text {nd }}$ approach } \\
\hline Class & $\begin{array}{c}\text { Sub- } \\
\text { classes }\end{array}$ & $\begin{array}{c}\text { Ob- } \\
\text { served } \\
\text { data }\end{array}$ & $\begin{array}{c}\text { MLE } \\
(\mathrm{M} 1 / \mathrm{M} 1+\mathrm{M} 2)\end{array}$ & $\begin{array}{c}\text { Processed } \\
\text { observed } \\
\text { data }\end{array}$ & $\begin{array}{c}\text { MAP } \\
(\mathrm{M} 1+\alpha-1) /(\mathrm{M} 1+\alpha+\mathrm{M} 2+\beta-2)\end{array}$ \\
\hline $\mathrm{R}$ & $\{\mathrm{A}, \mathrm{B}\}$ & 1 & $1 /(1+23)=0.042$ & 24 & $(24+1-1) /(24+1+0+1-2)=1.000$ \\
\hline $\mathrm{A}$ & $\{\mathrm{C}, \mathrm{D}\}$ & 1 & $1 /(1+23)=0.042$ & 8 & $(8+8-1) /(8+8+16+1-2)=0.484$ \\
\hline $\mathrm{B}$ & $\{\mathrm{E}, \mathrm{F}\}$ & 10 & $\begin{array}{l}10 /(10+14)= \\
0.417\end{array}$ & 15 & $(15+15-1) /(15+15+9+1-2)=0.763$ \\
\hline $\mathrm{C}$ & \{\} & 2 & $2 /(1+23)=0.083$ & 2 & $(2+2-1) /(2+2+22+7-2)=0.097$ \\
\hline $\mathrm{D}$ & $\{\mathrm{H}\}$ & 4 & $4 /(4+20)=0.167$ & 5 & $(5+5-1) /(5+5+19+4-2)=0.290$ \\
\hline $\mathrm{E}$ & \{\} & 1 & $1 /(1+23)=0.042$ & 1 & $(1+1-1) /(1+1+23+15-2)=0.026$ \\
\hline
\end{tabular}




\begin{tabular}{|c|c|c|c|c|c|}
\hline $\mathrm{F}$ & \{\} & 4 & $4 /(4+20)=0.167$ & 4 & $(4+4-1) /(4+4+20+12-2)=0.184$ \\
\hline $\mathrm{H}$ & \{\} & 1 & $1 /(1+23)=0.042$ & 1 & $(1+1-1) /(1+1+23+8-2)=0.032$ \\
\hline
\end{tabular}

\section{Discussion.}

The hayperparameter $\beta$ used to reflect the effect of the complement relation between class A and B. For example, the value of $\beta$ for class $\mathrm{H}$ is equals to 8 which means an instance which is belong to $\mathrm{H}$ workspace and not an instance of class $\mathrm{H}$, it only could be an instance of R, A, C or D because class A is complement of class B.

Figure 4.f shows the differences in marginal probabilities for all variables in example three when the semantic relations have been injected in the computation process and when they have been ignored. It shows that classes A, B and their subclasses have reflected the complement of semantic relation.

\section{Example four (union of logical relation).}

This example explains the union of logical relation. Figure 4.g shows that class E is the union of class $\mathrm{H}$ and $\mathrm{I}$. The semantic interpretation of this relation is that any instance of $\mathrm{H}$ or $\mathrm{I}$ is an instance of $\mathrm{E}$ as well. Table 11 explains the probabilistic constrains calculation process with and without considering the union of the semantic relation between classes in example four.

Table 11. Example four $1^{\text {st }}$ approach Vs $2^{\text {nd }}$ approach constraints calculations

\begin{tabular}{|c|c|c|c|c|c|}
\hline \multicolumn{2}{|c|}{ Example 4} & \multicolumn{2}{|c|}{$1^{\text {st }}$ approach } & \multicolumn{2}{|r|}{$2^{\text {nd }}$ approach } \\
\hline Class & $\begin{array}{l}\text { Sub- } \\
\text { classes }\end{array}$ & $\begin{array}{c}\text { Observed } \\
\text { data }\end{array}$ & $\begin{array}{c}\text { MLE } \\
(\mathrm{M} 1 / \mathrm{M} 1+\mathrm{M} 2)\end{array}$ & $\begin{array}{l}\text { cessed } \\
\text { observed } \\
\text { data }\end{array}$ & $\begin{array}{c}\text { MAP } \\
(\mathrm{M} 1+\alpha-1) /(\mathrm{M} 1+\alpha+\mathrm{M} 2+\beta-2)\end{array}$ \\
\hline $\mathrm{R}$ & $\{\mathrm{A}, \mathrm{B}\}$ & 1 & $1 /(1+22)=0.043$ & 23 & $(23+1-1) /(23+1+0+1-2)=1.000$ \\
\hline A & $\{C, D\}$ & 1 & $1 /(1+22)=0.043$ & 4 & $(4+1-1) /(4+1+19+1-2)=0.174$ \\
\hline $\mathrm{B}$ & $\{\mathrm{E}, \mathrm{F}\}$ & 1 & $1 /(1+22)=0.043$ & 18 & $(18+1-1) /(18+1+5+1-2)=0.783$ \\
\hline $\mathrm{C}$ & \{\} & 2 & $2 /(2+21)=0.087$ & 2 & $(2+1-1) /(2+1+21+1-2)=0.087$ \\
\hline $\mathrm{D}$ & \{\} & 1 & $1 /(1+22)=0.043$ & 1 & $(1+1-1) /(1+1+22+1-2)=0.043$ \\
\hline $\mathrm{E}$ & \{\} & 2 & $2 /(2+21)=0.087$ & 2 & $(2+15-1) /(2+15+21+8-2)=0.364$ \\
\hline $\mathrm{F}$ & $\{\mathrm{H}, \mathrm{I}\}$ & 2 & $2 /(2+21)=0.087$ & 15 & $(15+1-1)(15+1+8+1-2)=0.652$ \\
\hline $\mathrm{H}$ & V \{\}$^{\prime}$ & 5 & $5 /(5+18)=0.217$ & 5 & $(5+1-1) /(5+1+18+1-2)=0.217$ \\
\hline $\mathrm{I}$ & L\} & 8 & $8 /(8+15)=0.348$ & 8 & $(8+1-1) /(8+1+15+1-2)=0.348$ \\
\hline
\end{tabular}

\section{Discussion.}

Table 11 shows how the $\alpha$ hayperparameter utilized to inject the union relation in the estimation process. For example, the value of $\alpha$ for class $E$ is equal to 15 which is the sum of the instances for classes $\mathrm{H}$, I and E. This technique reflect the union relation which says that class $\mathrm{E}$ is the union of $\mathrm{H}$ and I. So, any instance of $\mathrm{H}$ and $\mathrm{I}$ is an instance of $\mathrm{E}$ as well.

Figure 4.h compares the marginal probability for all random variables in example four. It can be seen that the marginal probability for class $E$ which involve in 
the union relation has reflect the effect of this relation. Additionally, the effect of the superclass-subclass relations has been accommodated as well.

\section{Example five (intersection of logical relation).}

This example discusses the intersection of semantic relations. Figure 4.i shows that the class $\mathrm{H}$ is the intersection of class $\mathrm{E}$ and $\mathrm{F}$. So, semantically this relation means that any instance of class $\mathrm{H}$ is an instance of class $\mathrm{E}$ and $\mathrm{F}$. Table 12 compares between calculating the probabilistic constraints with and without considering the semantic relations for random variable in example five.

Table 12. Example five $1^{\text {st }}$ approach Vs $2^{\text {nd }}$ approach constraints calculations

\begin{tabular}{|c|c|c|c|c|c|}
\hline \multicolumn{2}{|c|}{ Example 5} & \multicolumn{2}{|c|}{$1^{\text {st }}$ approach } & \multicolumn{2}{c|}{$2^{\text {nd }}$ approach } \\
\hline $\begin{array}{c}\text { Clas } \\
\text { s }\end{array}$ & $\begin{array}{c}\text { Sub- } \\
\text { classes }\end{array}$ & $\begin{array}{c}\text { Ob- } \\
\text { served } \\
\text { data }\end{array}$ & $\begin{array}{c}\text { MLE } \\
\text { (M1/M1+M2) }\end{array}$ & $\begin{array}{c}\text { Pro- } \\
\text { cessed } \\
\text { observed } \\
\text { data }\end{array}$ & $\begin{array}{c}\text { MAP } \\
(\mathrm{M} 1+\alpha-1) /(\mathrm{M} 1+\alpha+\mathrm{M} 2+\beta-2)\end{array}$ \\
\hline $\mathrm{R}$ & $\{\mathrm{A}, \mathrm{B}\}$ & 1 & $1 /(1+28)=0.034$ & 29 & $(29+1-1) /(29+1+0+1-2)=1.000$ \\
\hline $\mathrm{A}$ & $\{\mathrm{C}, \mathrm{D}\}$ & 1 & $1 /(1+28)=0.034$ & 20 & $(20+1-1) /(20+1+9+1-2)=0.690$ \\
\hline $\mathrm{B}$ & $\{\mathrm{E}, \mathrm{F}\}$ & 1 & $1 /(1+28)=0.034$ & 8 & $(8+1-1) /(8+1+21+1-2)=0.276$ \\
\hline $\mathrm{C}$ & \{\} & 2 & $2 /(2+27)=0.069$ & 2 & $(2+1-1) /(2+1+27+1-2)=0.069$ \\
\hline $\mathrm{D}$ & $\{\mathrm{H}\}$ & 7 & $7 /(7+22)=0.241$ & 17 & $(17+1-1) /(17+1+12+1-2)=0.586$ \\
\hline $\mathrm{E}$ & \{\} & 2 & $2 /(2+27)=0.069$ & 2 & $(2+12-1) /(2+12+27+17-2)=0.232$ \\
\hline $\mathrm{F}$ & \{\} & 5 & $5 /(5+24)=0.172$ & 5 & $(5+15-1) /(5+15+24+14-2)=0.339$ \\
\hline $\mathrm{H}$ & \{\} & 10 & $10 /(10+19)=0.345$ & 10 & $(10+1-1) /(10+1+19+1-2)=0.345$ \\
\hline
\end{tabular}

\section{Discussion.}

Table 12 explains the effect of the intersection of logical relation in the constraints estimation process. It can be seen that the value of $\alpha$ for $\mathrm{E}$ and $\mathrm{F}$ class has reflect this relation. For example, the value of $\alpha$ for class $E$ is equal to 12 which is the sum of the instances for class $\mathrm{H}$ and $\mathrm{E}$. Likewise; the value of $\alpha$ for class $\mathrm{F}$ is equal to 15. Fig. 4.j shows the differences in marginal probabilities for all random variables when the semantic relation is integrated into the calculation process and when they have been ignored. The effect of intersection of relation is appeared in the marginal probability for $\mathrm{E}$ and $\mathrm{F}$. Furthermore, the marginal probability for all classes involved in the superclass-subclass relation has been altered as well. 

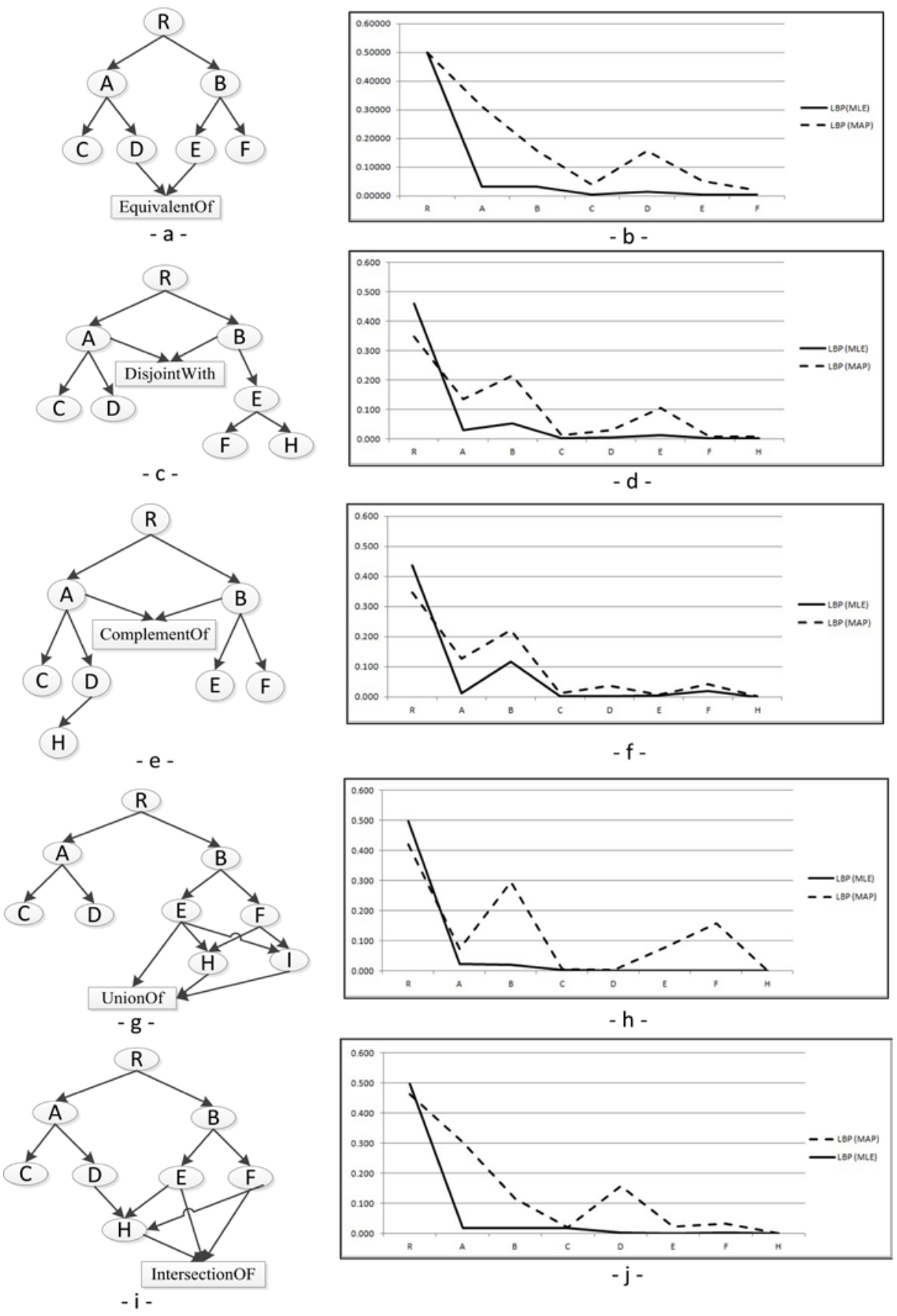

Fig. 4. BN structure with the associated marginal probabilities 


\section{Conclusions}

The integration of data semantics in the mining process is not a new concept. However, exploiting the semantic relation in LD via the mining process is a new research area. LD not only intertwines the data and it's semantic in one package, but also facilitates the integration of various data-sets from multiple domains.

One major drawback of the conventional mining algorithms is transforming the LD original format into a format understandable by the mining algorithm. Accordingly, they cannot benefit from the semantical and structural features of the LD. To this end, this paper proposed a semantic-aware Bayesian network model which exploits the semantic nature of LD and implicitly accommodates the data intelligence in the mining process. Furthermore, it does not change the original format of the LD. So, linking multiple data-sets is easily achievable.

The proposed approach consists of the following steps:

1. Convert the LD file into a BN which preserve the semantic of the original LD file.

2. Initialize the Conditional Probability Tables (CPT's) with default value.

3. Calculate set of probabilistic constraints using the concept of Maximum a Posterior Estimation (MAP) in such a way that reflect the semantic relation between nodes in the BN.

4. Approximate the CPT's initial values using the concept of Iterative Proportional Fitting Procedure (IPFP) and the set of probabilistic constraints estimated in the third step.

The proposed model has been tested using five sets of synthesized data. Each data-set highlight the significance of one logical relation. Initial results show that injecting the semantically aware probabilistic information into the Bayesian inference algorithm generates more realistic results.

Taking into account the fast accumulation of LD, this paper has investigated the suitability of Bayesian network for LD mining. Although synthesized data-set shows some promising results, the proposed model will be tested on real life data-set.

\section{References}

1. Fayyad, U., Piatetsky-Shapiro, G., Smyth, P.: From data mining to knowledge discovery in databases. AI Mag. 37-54 (1996).

2. Zhang, C., Zhang, S.: Association Rule Mining: Models and Algorithms. SpringerVerlag Berlin Heidelberg. XII, 244. (2002).

3. Cao, L., Yu, P.S., Zhang, C., Zhao, Y.: Domain driven data mining. Springer US.XVI, 248. (2010).

4. Cao, L.: Domain-driven data mining: Challenges and prospects. IEEE Trans. Knowl. Data Eng. 22, 755-769 (2010).

5. Sexton, M., Lu, S.: The challenges of creating actionable knowledge: an action research perspective. Constr. Manag. Econ. 2, 683-694 (2009).

6. Paul, R., Groza, T., Hunter, J., Zankl, A.: Semantic interestingness measures for dis- 
covering association rules in the skeletal dysplasia domain. J. Biomed. Semantics. 5, 8 (2014).

7. Dahan, H., Cohen, S., Rokach, L., Maimon, O.: Proactive Data Mining with Decision Trees. Springer New York (2014).

8. Antunes, C., Silva, A.: New Trends in Knowledge Driven Data Mining a position paper. Proc. 16th Int. Conf. Enterp. Inf. Syst. 346-351 (2014).

9. Bizer, C., Heath, T., Berners-Lee, T.: Linked data-the story so far. Int. J. Semant. Web Inf. Syst. 5, 1-22 (2009).

10. Quboa, Q.K., Saraee, M.: A State-of-the-Art Survey on Semantic Web Mining. Intell. Inf. Manag. 05, 10-17 (2013).

11. Ding, Z., Peng, Y., Pan, R.: BayesOWL: Uncertainty Modelling in Semantic Web Ontologies. Soft Comput. Ontol. Semant. Web. 204, 3-29 (2006).

12. Ma, Z.: Soft Computing in Ontologies and Semantic Web. Springer Sci. Bus. Media. (2007).

13. Sun, Y.: A Prototype Implementation of BayesOWL ,Diss. University of Mayryland Baltimore County, (2009).

14. Ding, Z.: BayesOWL, http://www.csee.umbc.edu/ ypeng/BayesOWL/index.html.

15. Ding, Z., Peng, Y.: A Bayesian approach to uncertainty modelling in OWL ontology, MARYLAND UNIV BALTIMORE DEPT OF COMPUTER SCIENCE AND ELECTRICAL ENGINEERING.,(2006).

16. Zhang, S., Sun, Y., Peng, Y., Wang, X.: A Practical Tool for Uncertainty in OWL Ontologies. Proc. 10th IASTED Int. Conf. 674, 235.

17. Koller, D., Friedman, N.: Probabilistic Graphical Models Principles and Techniques. MIT press (2009).

18. Jensen, F. V., Nielsen, T.D.: Bayesian Networks and Decision Graphs. Springer Science \& Business Media (2009).

19. Almond, R.G., Mislevy, R.J., Steinberg, L.S., Yan, D., Williamson, D.M.: Learning in Models with Fixed Structure. Bayesian Networks Educ. Assessment. Springer New York. 279-330 (2015).

20. Heinrich, G.: Parameter estimation for text analysis. Tech. Report, Fraunhofer IGD, Darmstadt, Ger. (2005).

21. Levy, R.: Probabilistic Models in the Study of Language , Diss,University of California, San Diego, (2012).

22. Fienberg, S.E.: An iterative procedure for estimation in contingency tables. Ann. Math. Statisitics. 907-917 (1970).

23. Cramer, E.: Probability measures with given marginals and conditionals: I-projections and conditional iterative proportional fitting. Stat. Decis. J. Stoch. Methods Model. 311 - 330 (2000).

24. Vomlel, J.: Methods of probabilistic knowledge integration, PhD Thesis, Czech Technical University, Faculty Of Electrical Engineering, (1999). 